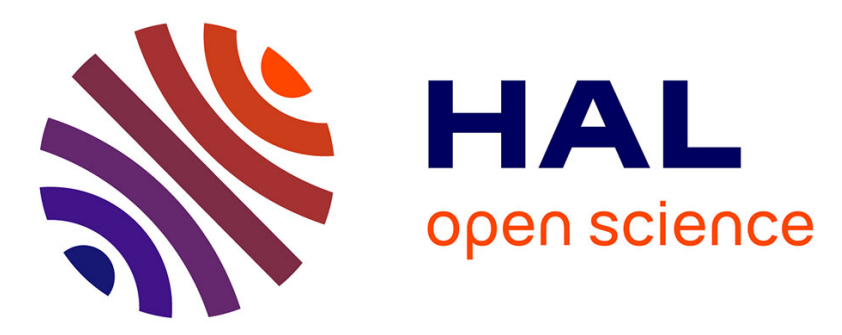

\title{
On the Use of Rényi Entropy for Optimal Window Size Computation in the Short-Time Fourier Transform
}

\author{
Sylvain Meignen, Marcelo Colominas, Duong-Hung Pham
}

\section{To cite this version:}

Sylvain Meignen, Marcelo Colominas, Duong-Hung Pham. On the Use of Rényi Entropy for Optimal Window Size Computation in the Short-Time Fourier Transform. 2020 IEEE International Conference on Acoustics, Speech and Signal Processing - ICASSP 2020, May 2020, Barcelone, Spain. pp.58305834, 10.1109/ICASSP40776.2020.9053392 . hal-02573674

\section{HAL Id: hal-02573674 \\ https://hal.science/hal-02573674}

Submitted on 2 Jun 2020

HAL is a multi-disciplinary open access archive for the deposit and dissemination of scientific research documents, whether they are published or not. The documents may come from teaching and research institutions in France or abroad, or from public or private research centers.
L'archive ouverte pluridisciplinaire HAL, est destinée au dépôt et à la diffusion de documents scientifiques de niveau recherche, publiés ou non, émanant des établissements d'enseignement et de recherche français ou étrangers, des laboratoires publics ou privés. 


\title{
On the Use of Rényi Entropy for Optimal Window Size Computation in the Short-Time Fourier Transform
}

Sylvain Meignen LJK, University Grenoble-Alpes

700 Avenue Centrale

38401 Saint-Martin-d'Hères, France

sylvain.meignen@univ-grenoble-alpes.fr Marcelo Colominas IBB, CONICET

Ruta Prov. 11 Km. 10, Oro Verde,

Entre Ríos, Argentina

macolominas@conicet.gov.ar Duong-Hung Pham IRIT Laboratory

118 Route de Narbonne,

31062 Toulouse, France

duong-hung.pham@irit.fr

\begin{abstract}
In this paper, we investigate the determination of an optimal window length associated with the computation of the short time Fourier transform of multicomponent signals. In recent years the Rényi entropy has been widely used for that purpose, but the understanding of the significance of the obtained minimum in relation with the studied signal is still partial. In this paper, we explain in what way the window minimizing the Rényi entropy reflects the modulation of the modes making up the signal, and in which circumstances to use such a window is actually relevant.
\end{abstract}

\section{Index Terms}

Short time Fourier transform; multicomponent signals; Rényi entropy

\section{INTRODUCTION}

The study of multicomponent signals (MCSs) using the short-time Fourier transform (STFT) is very common in the time-frequency (TF) literature. These signals are of great interest to the signal processing community because of their ability to accurately represent non-stationary signals arising from a wide range of applications e.g., audio recordings, structural stability [1], [2], or physiological signals [3]. However, the effectiveness of the STFT on 
these signals is constrained by the choice of the window, since the Heisenberg uncertainty principle limits both the adaptivity and the readability of this TF representation. Many different techniques known under the generic term of reassignment methods (RMs), initially proposed by Kodera et al. [4] in the 1970s and then further developed in [5], aimed at compensating for the uncertainty principle by reallocating the coefficient in the TF plane.

Nevertheless, in spite of these efforts, the quality of the reassignment process is tightly related to the choice of window, for which a very common choice is to consider the one associated with the minimal Rényi entropy [6] [7]. In practice such a choice usually leads to good results and an thorough analysis of the Rényi entropy of Gaussian logons was carried out [8] [9]. Such an entropy also proved to be an interesting tool for mode counting [10]. However, the analysis of the minimum of the Rényi entropy in relation with the modulation of the modes contained in the signal has never been carried out and is the subject of the present paper. After having introduced useful notation, we investigate the significance of the minimal Rényi entropy for pure harmonic signals, parallel linear chirps, and more general signals, to conclude how the minimal Rényi entropy is connected with the modulation of the modes.

\section{DEFINITIONS AND NotATion}

Prior to starting, we recall useful notation and definitions, that will be used throughout the paper. Consider a signal $f \in L^{1}(\mathbb{R})$, its Fourier transform corresponds to:

$$
\widehat{f}(\xi)=\mathcal{F}\{f\}(\xi)=\int_{\mathbb{R}} f(t) e^{-i 2 \pi \xi t} d t,
$$

and its short-time Fourier transform (STFT) is defined using any sliding window $g \in L^{\infty}(\mathbb{R})$ by:

$$
\begin{aligned}
V_{f}^{g}(t, \xi) & =\int_{\mathbb{R}} f(\tau) g(\tau-t) e^{-2 i \pi \xi(\tau-t)} d \tau \\
& =\int_{\mathbb{R}} f(t+\tau) g(\tau) e^{-2 i \pi \xi \tau} d \tau .
\end{aligned}
$$

In this paper, we will intensively study multicomponent signals (MCSs) defined as a superimposition of AM-FM components or modes:

$$
f(t)=\sum_{k=1}^{K} f_{k}(t) \quad \text { with } f_{k}(t)=A_{k}(t) e^{i 2 \pi \phi_{k}(t)},
$$

for some finite $K \in \mathbb{N}, A_{k}(t)$ and $\phi_{k}^{\prime}(t)$ being respectively the instantaneous amplitude (IA) and frequency (IF) $f_{k}$ satisfying: $A_{k}(t)>0, \phi_{k}^{\prime}(t)>0$ and $\phi_{k+1}^{\prime}(t)>\phi_{k}^{\prime}(t)$ for all $t$.

We also assume that $A_{k}$ is differentiable with $A_{k}^{\prime}$ small compared with $\phi_{k}^{\prime}$, that the modes are separated with resolution $\Delta$, i.e. for all time $t$,

$$
\forall 1 \leq k \leq K-1, \phi_{k+1}^{\prime}(t)-\phi_{k}^{\prime}(t)>2 \Delta
$$

In practice, one has to deal with finite length $f$, typically defined, without loss of generality, on the interval $[0,1]$, discretized into $f\left(\frac{n}{L}\right)_{n=0, \cdots, L-1}$, and $g$ supported on $\left[-\frac{M}{L}, \frac{M}{L}\right]$ with $M<L / 2$. The STFT of $f$ is then computed 
as follows:

$$
\begin{aligned}
V_{f}^{g}(t, \xi) & =\int_{-\frac{M}{L}}^{\frac{M}{L}} f(t+\tau) g(\tau) e^{-2 i \pi \tau \xi} d \tau \\
& \approx \frac{1}{L} \sum_{n=-M}^{M} f\left(t+\frac{n}{L}\right) g\left(\frac{n}{L}\right) e^{-i 2 \pi \frac{n}{L} \xi},
\end{aligned}
$$

which equals at time $\frac{m}{L}$ and frequency $\frac{k L}{N}$ to

$$
V_{f}^{g}[m, k]:=\sum_{n=-M}^{M} f\left(\frac{(m+n)}{L}\right) g\left(\frac{n}{L}\right) \frac{e^{-i 2 \pi \frac{n k}{N}}}{L},
$$

for some $N \geq 2 M+1$ and then $0 \leq k \leq N-1$. In practical situations, $M$ is fixed depending on $N$ through $M=\left\lfloor\frac{N-1}{2}\right\rfloor$.

Let us now recall the definition of the Rényi entropy computed on the magntitude of the STFT, defined in the continuous time and frequency context on $R=[0,1] \times[0, L][8]$ :

$$
=\frac{1}{1-\alpha} \log _{2}\left(\int_{R}\left(\frac{\left|V_{f}^{g}(t, \xi)\right|}{\int_{R}\left|V_{f}^{g}\left(t^{\prime}, \xi^{\prime}\right)\right| d t^{\prime} d \xi^{\prime}}\right)^{\alpha} d t d \xi\right) .
$$

Discretizing $R$ along the time axis by a factor of $\frac{1}{L}$ and along the frequency axis by a factor of $\frac{L}{N}$, one obtains a grid $G$. Each value of the discrete STFT can be associated with a TF square of size $\frac{1}{L} \times \frac{L}{N}=\frac{1}{N}$, and thus we may discretize the Rényi entropy into:

$$
:=\frac{1}{1-\alpha} \log _{2}\left(\sum_{(n, k) \in G}\left(\frac{\left|V_{f}^{g}[n, k]\right|}{\sum_{\left(n^{\prime}, k^{\prime}\right) \in G}\left|V_{f}^{g}\left[n^{\prime}, k^{\prime}\right]\right|}\right)^{\alpha}\right)^{\alpha}[f]
$$

As explained in the next section, Rényi entropies are the basic tools for the determination of optimal window length.

\section{Optimal Window Length Determination Based on RÉnyi Entropy and Mode Modulation}

A crucial aspect to obtain meaningful STFT is the determination of an appropriate window length supported on $\left\{-\left\lfloor\frac{N-1}{2}\right\rfloor, \cdots,\left\lfloor\frac{N-1}{2}\right\rfloor\right\}$. For that purpose, a very popular approach consisting of computing the minimum of the Rényi entropy on the magnitude of the STFT and then define the optimal filter as the one realizing this minimum [6] [11]. The meaning of this minimum with respect to the studied MCS being however unclear, we aim, in what follows, to analyze this minimum to put forward its relation with the modulation of the modes making up the signal.

\section{A. Determining the Optimal Window for MCS Made of Purely Harmonic modes Using Rényi Entropy}

The first study we carry out is on a pure tone signal $f(t)=e^{2 i \pi \omega_{1} t}$, for $t \in[0,1]$, for which $\left|V_{f}^{g}(t, \xi)\right|=$ $\left|\hat{g}\left(\xi-\omega_{1}\right)\right|$. Now assume the Gaussian window $g(t):=g_{s}(t)=e^{-\pi \frac{t^{2}}{\sigma_{s}^{2}}}$ is used, then we may write that $\left|V_{f}^{g_{s}}(t, \xi)\right|=$ 
$\sigma_{s} e^{-\pi \sigma_{s}^{2}\left(\xi-\omega_{1}\right)^{2}}$, so that:

$$
\begin{array}{r}
=\frac{1}{1-\alpha} \log _{2}\left(\int_{R}\left(\frac{\sigma_{s} e^{-\pi \sigma_{s}^{2}\left(\xi-\omega_{1}\right)^{2}}}{\int_{R} \sigma_{s} e^{-\pi \sigma_{s}^{2}\left(\xi^{\prime}-\omega_{1}\right)^{2} d t^{\prime} d \xi^{\prime}}}\right)^{\alpha} d t d \xi\right) \\
H_{\alpha, g_{s}}^{R}(f) \\
\approx \frac{1}{1-\alpha} \log _{2}\left(\int_{R}\left(\frac{e^{-\pi\left(\xi-\sigma_{s} \omega_{1}\right)^{2}}}{\int_{R} e^{-\pi\left(\xi^{\prime}-\sigma_{s} \omega_{1}\right)^{2}} d t^{\prime} d \xi^{\prime}}\right)^{\alpha} d t d \xi\right) \\
-\log _{2}\left(\sigma_{s}\right)
\end{array}
$$

the approximation being valid only if:

$$
e^{-\pi \sigma_{s}^{2}\left(L-\omega_{1}\right)^{2}} \leq \varepsilon \text { if } \sigma_{s} \leq 1
$$

for a small $\varepsilon$. We will see a bit later that only $\sigma_{s} \leq 1$ has to be considered. Assuming this condition is satisfied, we may write

$$
\begin{aligned}
H_{\alpha, g_{s}}^{R}(f) & \approx H_{\alpha, g_{1}}^{R}\left(f\left(\sigma_{s} .\right)\right)-\log _{2}\left(\sigma_{s}\right) \\
& \approx H_{\alpha, g_{1}}^{R}(f)-\log _{2}\left(\sigma_{s}\right)
\end{aligned}
$$

provided, when $\sigma_{s} \leq 1, e^{-\pi \sigma_{s}^{2} \omega_{1}^{2}} \leq \varepsilon$, meaning

$$
\frac{\sqrt{-\frac{\log (\varepsilon)}{\pi}}}{\omega_{1}} \leq \sigma_{s} .
$$

When (11) is satisfied, the Rényi entropy is a decreasing function with respect to $\sigma_{s}$. Note that condition (12) is always stronger than (10) (because it means $2 \omega_{1} \leq L$, which is true due to Nyquist principle).

In practice, one also has to take into account that the Gaussian window is truncated, and, to minimize truncation errors, an upper bound $\sigma_{\max }$ for $\sigma_{s}$ has to be imposed, typically $e^{-\pi \frac{\left\lfloor\frac{N-1}{2}\right\rfloor^{2}}{\sigma_{\max }^{2} L^{2}}}=\varepsilon$, meaning:

$$
\sigma_{s} \leq \sigma_{\max }=\frac{\left\lfloor\frac{N-1}{2}\right\rfloor}{L} \sqrt{\frac{-\pi}{\log (\varepsilon)}}
$$

This upper bound for $\sigma_{s}$ being always much lower than one this justifies a posteriori that $\sigma_{s}>1$ need not be considered. As a first numerical illustration we consider $\omega_{1}=400, L=4096, N=512$ and $\varepsilon=10^{-3}$, leading $\sigma_{\max }=0.042$, while (12) leads $\sigma_{s} \geq \frac{\sqrt{-\frac{\log (\varepsilon)}{\pi}}}{400}=0.0037$. The interval of interest for $\sigma_{s}$ is thus $I=[0.0037,0.042]$. Now, consider the signal $f$ made of these two pure tones signals, namely $f(t)=f_{1}(t)+f_{2}(t)=2 e^{2 i \pi 400 t}+2 e^{2 i \pi 800 t}$. Assuming the modes are slightly interfering in the TF plane, the constraints (12) and (13) for each mode lead to the same interval of interest as in the single mode case. Then, and as remarked in [8] for two Gaussian logons, one can easily prove that the Rényi entropy should satisfy:

$$
H_{\alpha, g_{s}}^{R}\left(f_{1}+f_{2}\right) \approx H_{\alpha, g_{s}}^{R}\left(f_{1}\right)+1
$$

this property being also true for the discretized version of the Rényi entropy $H_{\alpha, g_{s}}^{G}$. However, if the modes are moved closer, namely if one changes $f_{2}$ into $2 e^{2 i \pi 600 t}$ and then into $2 e^{2 i \pi 450 t}$, for small values for $\sigma_{s}$ the separation 


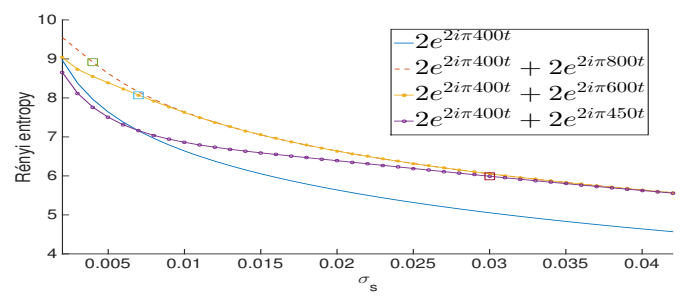

(a)

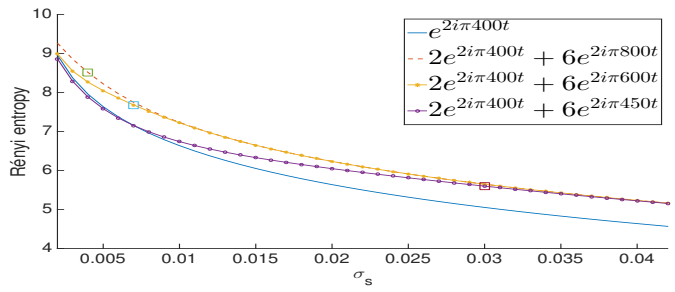

(b)

Fig. 1. (a): computation of the Rényi entropy as a function of $\sigma_{s}$ when $f_{1}(t)=2 e^{2 i \pi 400 t}$ and $f_{2}(t)=2 e^{2 i \pi 800 t}$, the plain line is for $f_{1}$ only and the dashed line corresponds to $f_{1}+f_{2}$, the line marked with circle corresponds to $f_{2}(t)=2 e^{2 i \pi 600 t}, f_{1}$ being unchanged, and the line marked with stars to $f_{2}(t)=2 e^{2 i \pi 450 t}, f_{1}$ being still unchanged, the squares represent the entropies at $\sigma_{s}=\frac{\sqrt{-\frac{\log (\varepsilon)}{\pi}}}{\min \left(\omega_{1}, \omega_{2}-\omega_{1}\right)}$; (b): same as (a) except that the amplitude of $f_{2}$ is 6 instead of 2 .

of the modes is not achieved in the sense that (14) is not satisfied. One can however still define an interval for $\sigma_{s}$ in which the modes can be considered as separated in the TF plane replacing (12) by :

$$
\frac{\sqrt{-\frac{\log (\varepsilon)}{\pi}}}{\min \left(\omega_{1}, \frac{\omega_{2}-\omega_{1}}{2}\right)} \leq \sigma_{s},
$$

where $\omega_{2}$ is the frequency of the second mode, the definition of $\sigma_{\max }$ remaining unchanged. This lower bound can be interpreted as follows: the STFT of the two mode signal can be considered as that of two separated modes if at frequency $\frac{\omega_{1}+\omega_{2}}{2}$ the second mode is not interfering with the first one, i.e. namely $e^{-\pi \sigma_{s}\left(\frac{\omega_{1}-\omega_{2}}{2}\right)} \leq \varepsilon$, which leads to $(15)$.

In Fig. 1 (a), we display the Rényi entropy (with $\alpha=3$ ) associated with the single mode signal, i.e. $f_{1}$, or the two mode signal, i.e. $f_{1}+f_{2}$, when $\sigma_{s}$ varies in $I=[0.0037,0.042]$ and when mode $f_{2}$ varies, $f_{1}$ being fixed. Condition (15) implies that $\sigma_{s}$ has to be larger that 0.0148 (resp. 0.0593) for the case associated with the line marked with stars (resp. circle) in Fig. 1 (a). A closer look at Fig. 1 (a) tells us that the separation condition (14) is satisfied even when condition (15) is not, that is when the modes are slightly interfering. In this regard, to assume that at frequency $\omega_{1}$, the STFT of the second mode is negligible, meaning replacing $\frac{\omega_{2}-\omega_{1}}{2}$ by $\omega_{2}-\omega_{1}$ is sufficient to fulfill condition (14) (we have plotted on Fig. 1 squares to show this lower bound for $\sigma_{s}$ ). From this study, the interval of interest for $\sigma_{s}$ becomes $\left[\frac{\sqrt{-\frac{\log (\varepsilon)}{\pi}}}{\min \left(\omega_{1}, \omega_{2}-\omega_{1}\right)}, \frac{\left\lfloor\frac{N-1}{2}\right\rfloor}{L} \sqrt{\frac{-\pi}{\log (\varepsilon)}}\right]$, and the minimum for the Rényi entropy corresponds to the larger value for $\sigma_{s}$ in that interval.

More generally, when one considers a sum of $K$ pure tones with possibly different amplitudes, the lower bound 
for $\sigma_{s}$ rewrites:

$$
\frac{\sqrt{-\frac{\log (\varepsilon)}{\pi}}}{\min \left(\omega_{1}, 2 \Delta\right)} \leq \sigma_{s}
$$

The separation condition (14) no longer holds and has then to be replaced by, $H_{\alpha, g_{s}}^{R}(f) \approx H_{\alpha, g_{s}}^{R}\left(f_{1}\right)+C\left(f_{1}, \cdots, f_{K}\right)$, where $C\left(f_{1}, \cdots, f_{K}\right)$ is a function varying between 0 and 1 which is maximal when all the components have equal amplitudes [9]. As a simple illustration, we display in Fig. 1 (b), the Rényi entropy of two modes with different amplitudes for which we clearly see that $C\left(f_{1}, f_{2}\right)<1$ but remains constant when $\sigma_{s}$ is appropriately chosen (note that a simple computation leads to $C\left(f_{1}, f_{2}\right)=\frac{1}{1-\alpha} \log _{2}\left(\frac{A_{1}^{\alpha}+A_{2}^{\alpha}}{\left(A_{1}+A_{2}\right)^{\alpha}}\right)$ ). As previously, the interval of interest where the separation actually occurs is $\left[\frac{\sqrt{-\frac{\log (\varepsilon)}{\pi}}}{\min \left(\omega_{1}, 2 \Delta\right)}, \frac{\left\lfloor\frac{N-1}{2}\right\rfloor}{L} \sqrt{\frac{-\pi}{\log (\varepsilon)}}\right]$, and is not a function of the amplitude (see the squares plotted on Fig. 1 (b) corresponding to the lower bound for $\sigma_{s}$ ), and the minimum entropy is always attains for the larger values for $\sigma_{s}$ in that interval.

\section{B. Computing the Optimal Window Length on Parallel Linear Chirps}

Now let us consider the case of parallel linear chirps. It was remarked that if $f$ is a linear chirp with constant amplitude A, one has (still assuming $\left.g_{s}(t)=e^{-\pi \frac{t^{2}}{\sigma_{s}^{2}}}\right)[12]$ :

$$
\left|V_{f}^{g}(t, \xi)\right|=A \sigma_{s}\left(1+\sigma_{s}^{4} \phi^{\prime \prime}(t)^{2}\right)^{-\frac{1}{4}} e^{-\pi \frac{\sigma_{s}^{2}\left(\xi-\phi^{\prime}(t)\right)^{2}}{1+\sigma_{s}^{4} \phi^{\prime \prime}(t)^{2}}} .
$$

Then, carrying out the same computation as for the pure tone signal, putting $\tilde{\sigma}_{s}=\frac{\sigma_{s}}{\sqrt{1+\sigma_{s}^{4} \phi^{\prime \prime}(t)^{2}}}$, one can rewrite the Rényi entropy of a linear chirp as:

$$
\begin{array}{r}
H_{\alpha, g_{s}}(f) \\
=\frac{1}{1-\alpha} \log _{2}\left(\int_{R}\left(\frac{e^{-\pi \tilde{\sigma}_{s}^{2}\left(\xi-\phi^{\prime}(t)\right)^{2}}}{\int_{R} e^{-\pi \tilde{\sigma}_{s}^{2}\left(\xi^{\prime}-\phi^{\prime}(t)\right)^{2}} d t^{\prime} d \xi^{\prime}}\right)^{\alpha} d t d \xi\right) \\
\approx \frac{1}{1-\alpha} \log _{2}\left(\int_{R}\left(\frac{e^{-\pi\left(\xi-\tilde{\sigma}_{s} \phi^{\prime}(t)\right)^{2}}}{\int_{R} e^{-\pi\left(\xi^{\prime}-\tilde{\sigma}_{s} \phi^{\prime}(t)\right)^{2}} d t^{\prime} d \xi^{\prime}}\right)^{\alpha} \tilde{\sigma}_{s}^{\alpha-1} d t d \xi\right), \\
=\frac{1}{1-\alpha} \log _{2}\left(\int_{R}\left(\frac{e^{-\pi\left(\xi-\tilde{\sigma}_{s} \phi^{\prime}(t)\right)^{2}}}{\int_{R} e^{-\pi\left(\omega^{\prime}-\tilde{\sigma}_{s} \phi^{\prime}(t)\right)^{2}} d t^{\prime} d \xi^{\prime}}\right)^{\alpha} d t d \xi\right) \\
-\log _{2}\left(\tilde{\sigma}_{s}\right),
\end{array}
$$

the approximation being valid if forall $t, e^{-\pi \tilde{\sigma}_{s}^{2}\left(L-\phi^{\prime}(t)\right)^{2}} \leq \varepsilon$, because $\sigma_{s}$ will be, as in the previous case, lower than 1 . We may then write:

$$
H_{\alpha, g_{s}}^{R}(f) \approx H_{\alpha, g_{1}}^{R}(f)-\log _{2}\left(\tilde{\sigma}_{s}\right)
$$

if, for each $t$

$$
\frac{\sqrt{-\frac{\log (\varepsilon)}{\pi}}}{\phi^{\prime}(t)} \leq \frac{\sigma_{s}}{\sqrt{1+\sigma_{s}^{4} \phi^{\prime \prime}(t)^{2}}} \leq \sigma_{\max },
$$

$\sigma_{\max }$ being defined in the previous section. A similar computation could be carried out for the dicrete version of Rényi entropy. When these conditions are satisfied, $H_{\alpha, g_{s}}^{R}(f)$ decreases (resp. increases) when $\tilde{\sigma}_{s}$ increases (resp. 
decreases). Computing the derivative of $\tilde{\sigma}_{s}$, one gets:

$$
\frac{1}{\sqrt{1+\sigma_{s}^{4} \phi^{\prime \prime}(t)^{2}}}+\sigma_{s}\left(-\frac{1}{2} \frac{4 \sigma_{s}^{3} \phi^{\prime \prime}(t)^{2}}{\left(1+\sigma_{s}^{4} \phi^{\prime \prime}(t)^{2}\right)^{\frac{3}{2}}}\right),
$$

which is null provided $\sigma_{s}^{4} \phi^{\prime \prime}(t)^{2}=1$, and the Rényi entropy decreases if $\sigma_{s} \leq \frac{1}{\sqrt{\phi^{\prime \prime}(t)}}$ and increases otherwise.

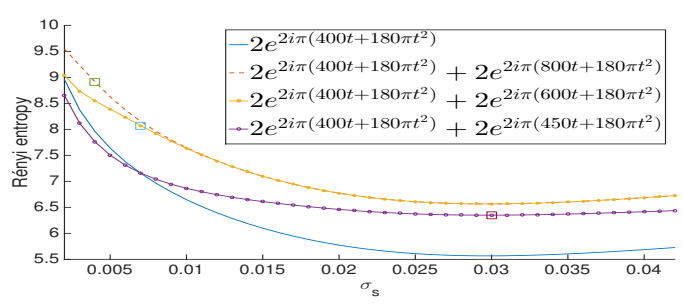

(a)

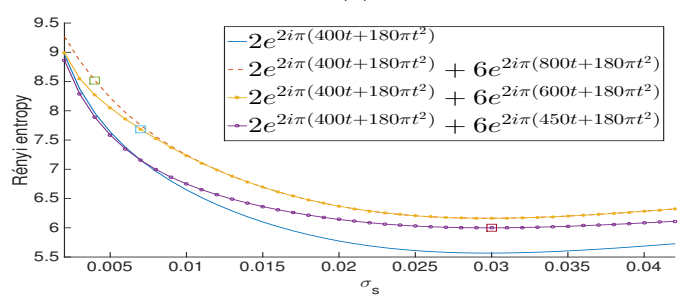

(b)

Fig. 2. (a): Computation of the Rényi entropy as a function of $\sigma_{s}$, with $N=512$, when $f_{1}(t)=2 e^{2 i \pi 400 t+180 \pi t^{2}}$ and $f_{2}(t)=$ $2 e^{2 i \pi 800 t+180 \pi t^{2}}$, the plain line is for $f_{1}$ only and the dashed line corresponds to $f_{1}+f_{2}$, then $f_{2}$ is replaced by $2 e^{2 i \pi 600 t+180 \pi t^{2}}$ (plain line marked with stars) and then by $2 e^{2 i \pi 450 t+180 \pi t^{2}}$ (plain line marked with circles), the squares correspond to the value of the Rényi entropy at $\sigma_{s}=\frac{\sqrt{-\frac{\log (\varepsilon)}{\pi}}}{\min \left(\phi_{1}^{\prime}(t), \phi_{2}^{\prime}(t)-\phi_{1}^{\prime}(t)\right)}$.; (b): same as (a) except the amplitude of the second mode is 6 .

We check that this is actually the case, by computing the Rényi entropy on $f=f_{1}$ or $f=f_{1}+f_{2}$, with $f_{1}(t)=2 e^{2 i \pi 400 t+180 \pi t^{2}}$ and $f_{2}(t)=2 e^{2 i \pi 800 t+180 \pi t^{2}}$. The results, displayed in Fig. 2 (a), confirm that the Rényi entropy passes through a minimum when $\sigma_{s}=\frac{1}{\sqrt{\phi_{1}^{\prime \prime}(t)}}$, when $f=f_{1}$ but also when $f=f_{1}+f_{2}$ since the two chirps are parallel and sufficiently far apart. Furthermore, the entropy of the two mode signal is again almost parallel to that of the single mode signal when, as in the pure harmonics case, $\sigma_{s}$ is larger than $\frac{\sqrt{-\frac{\log (\varepsilon)}{m i n}\left(\phi_{1}^{\prime}(t), \phi_{2}^{\prime}(t)-\phi_{1}^{\prime}(t)\right)}}{\text {. }}$.

To check these points, we consider two other two mode signals by moving $f_{2}$ closer to $f_{1}$ in the TF plane, the two chirps $f_{1}$ and $f_{2}$ remaining parallel, namely by considering $f_{2}(t)=2 e^{2 i \pi\left(600 t+180 \pi t^{2}\right)}$ or $f_{2}(t)=2 e^{2 i \pi\left(450 t+180 \pi t^{2}\right)}$, and plot a square on the graphs of Fig. 2 (a) when the Rényi entropy is evaluated at $\sigma_{s}=\frac{\sqrt{-\frac{\log (\varepsilon)}{\pi}}}{\min \left(\phi_{1}^{\prime}(t), \phi_{2}^{\prime}(t)-\phi_{1}^{\prime}(t)\right)}$. For $\sigma_{s}$ larger than this lower bound the Rényi entropy of the two mode signal is parallel to that in the single mode case. We also check that the minimum of the Rényi entropy is still connected to the chirp rate in all these cases. Finally, the Rényi entropy associated with several parallel linear chirps with different amplitudes is also considered, and the results of Fig. 2 (b), reveal no significant difference with the case of modes with the same amplitude. 


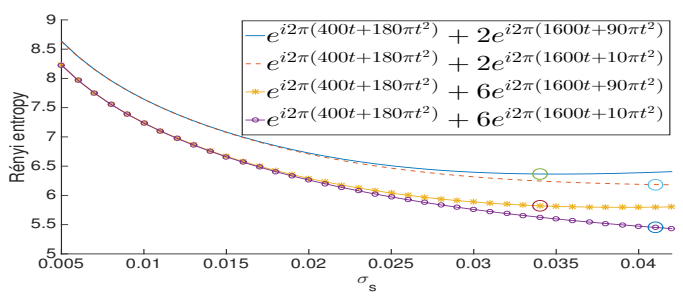

(a)

Fig. 3. (a): Rényi entropy made of two linear chirps, $f_{1}(t)=2 e^{i 2 \pi\left(400 t+180 \pi t^{2}\right)}$ and $f_{2}(t)=2 e^{i 2 \pi\left(1600 t+90 \pi t^{2}\right)}$ (plain line) or $f_{2}(t)=$ $2 e^{i 2 \pi\left(1600 t+10 \pi t^{2}\right)}$ (dashed line). Then, $f_{2}$ is replaced by $6 e^{i 2 \pi\left(1600 t+90 \pi t^{2}\right)}$ (plain line marked with stars) and then by $6 e^{i 2 \pi\left(1600 t+10 \pi t^{2}\right)}$ (plain line marked with circles). The Rényi entropy at $\sigma_{s}=\frac{1}{\sqrt{\frac{\phi_{1}^{\prime \prime}(t)+\phi_{2}^{\prime \prime}(t)}{2}}}$ is also plotted in each case (circle).

\section{Behavior of the Rényi Entropy on Non Parallel Linear Chirps}

In this section, we investigate the following conjecture. When the Rényi entropy associated with two linear chirps of possibly different amplitudes has a minimum inside the interval of interest, this minimum corresponds to $\sigma_{s}=\frac{1}{\sqrt{\frac{\phi_{1}^{\prime \prime}(t)+\phi_{2}^{\prime \prime}(t)}{2}}}$. As an illustration we consider the two mode signal with modes $f_{1}(t)=2 e^{2 i \pi\left(400 t+180 \pi t^{2}\right)}$ and $f_{2}(t)=2 e^{2 i \pi\left(1600 t+90 \pi t^{2}\right)}$ or $f_{2}(t)=2 e^{2 i \pi\left(1600 t+10 \pi t^{2}\right)}$, for which we compute the Rényi entropies displayed in Fig. 3 along with the value of the Rényi entropy at $\sigma_{s}=\frac{1}{\sqrt{\frac{\phi_{1}^{\prime \prime}(t)+\phi_{2}^{\prime \prime}(t)}{2}}}$ in each case (circles in Fig. 3). We see that in the first case, the minimum actually exists and corresponds to what we conjecture, while in the second case, $f_{2}$ being only slightly modulated the Rényi entropy does not pass through a minimum in the interval of interest, and the minimum of the optimal filter corresponds to the largest one compatible with the frequency resolution. By changing the amplitude of the second mode we see that the conclusions are the same with chirps with different amplitudes.

\section{CONCLUSiON}

In this paper, we have investigated the significance of the minimum of the Rényi entropy for the determination of the optimal window length for the computation of the short-time Fourier transform of multicomponent signals. Simple examples have shown us that when that minimum exists in some interval of interest, it reflects the modulation of the studied modes. For parallel linear chirps, the relation between window length and modulation can be derived analytically, but a more complex relation had to be conjectured for non parallel linear chirps. Future work should involve the analysis of the significance of the minimum of the Rényi entropy when the signal is made of more than two modes and then investigate local Rényi entropy to deal with more complicated modulated signals than linear chirps.

\section{REFERENCES}

[1] M. Costa, A. A. Priplata, L. A. Lipsitz, Z. Wu, N. E. Huang, A. L. Goldberger, and C.-K. Peng, "Noise and poise: Enhancement of postural complexity in the elderly with a stochastic-resonance-based therapy," Europhysics Letters (EPL), vol. 77, no. 6, p. 68008, Mar 2007. 
[2] D. A. Cummings, R. A. Irizarry, N. E. Huang, T. P. Endy, A. Nisalak, K. Ungchusak, and D. S. Burke, "Travelling waves in the occurrence of dengue haemorrhagic fever in Thailand," Nature, vol. 427, no. 6972, pp. 344 -347, Jan 2004.

[3] C. L. Herry, M. Frasch, A. J. Seely, and H.-T. Wu, "Heart beat classification from single-lead ecg using the synchrosqueezing transform," Physiological Measurement, vol. 38, no. 2, pp. 171-187, 2017.

[4] K. Kodera, R. Gendrin, and C. Villedary, "Analysis of time-varying signals with small bt values," IEEE Transactions on Acoustics, Speech, and Signal Processing, vol. 26, no. 1, pp. 64-76, Feb 1978.

[5] F. Auger and P. Flandrin, "Improving the readability of time-frequency and time-scale representations by the reassignment method," IEEE Transactions on Signal Processing, vol. 43, no. 5, pp. 1068-1089, 1995.

[6] S. Meignen, D.-H. Pham, and S. McLaughlin, "On demodulation, ridge detection, and synchrosqueezing for multicomponent signals," IEEE Transactions on Signal Processing, vol. 65, no. 8, pp. 2093-2103, 2017.

[7] D.-H. Pham and S. Meignen, "High-order synchrosqueezing transform for multicomponent signals analysis - with an application to gravitational-wave signal," IEEE Transactions on Signal Processing, vol. 65, no. 12, pp. 3168-3178, June 2017.

[8] R. G. Baraniuk, P. Flandrin, A. J. Janssen, and O. J. Michel, "Measuring time-frequency information content using the rényi entropies," IEEE Transactions on Information theory, vol. 47, no. 4, pp. 1391-1409, 2001.

[9] O. Michel, R. G. Baraniuk, and P. Flandrin, "Time-frequency based distance and divergence measures," in Proceedings of IEEE-SP International Symposium on Time-Frequency and Time-Scale Analysis. IEEE, 1994, pp. 64-67.

[10] N. Saulig, N. Pustelnik, P. Borgnat, P. Flandrin, and V. Sucic, "Instantaneous counting of components in nonstationary signals," in 21st European Signal Processing Conference (EUSIPCO 2013). IEEE, 2013, pp. 1-5.

[11] S. Meignen and D.-H. Pham, "Retrieval of the modes of multicomponent signals from downsampled short-time fourier transform," IEEE Transactions on Signal Processing, vol. 66, no. 23, pp. 6204-6215, 2018.

[12] R. Behera, S. Meignen, and T. Oberlin, "Theoretical analysis of the second-order synchrosqueezing transform," Applied and Computational Harmonic Analysis, vol. 45, no. 2, pp. 379-404, 2018. 